\title{
Diferencias en los motivos hacia la actividad física de los universitarios según nivel de actividad y su relación con la satisfacción del servicio deportivo universitario Differences in motives toward physical activity regarding physical activity level and its relationship with satisfaction about the university sport service
}

\author{
*Miguel Corbí *Carmen Palmero-Cámara, **Alfredo Jiménez-Palmero
}

*Universidad de Burgos (España); **Kedge Business School (Talence, Francia)

Resumen. El objetivo de este trabajo ha sido analizar los motivos hacia la actividad físico-deportiva de una muestra representativa de estudiantes de la Universidad de Burgos, y conocer la relación de estos con el servicio deportivo de la Universidad. 366 estudiantes (127 hombres y 239 mujeres), con una edad media de 21.29 años, respondieron la escala validada sobre motivos hacia la actividad física Goal Content Exercise Questionnaire (GCEQ), y un cuestionario sobre niveles de actividad físico-deportiva y participación en las actividades propuestas por el servicio deportivo de la Universidad. A partir de la interpretación de los datos se determinó que el $48.6 \%$ de los estudiantes realiza, al menos, una actividad físico-deportiva semanalmente, y que el motivo principal es el cuidado de salud seguido del desarrollo de las habilidades, no encontrando motivos acerca de aspectos sociales. Menos de una cuarta parte del alumnado ha utilizado el servicio deportivo universitario. El aspecto peor valorado con respecto a este servicio fue la compatibilidad de horarios. Se concluye que el alumnado universitario manifiesta bajos niveles de actividad física, tanto en el ámbito formativo como fuera de él. Los motivos de estos hacia la actividad físico-deportiva no muestran grandes diferencias con respecto a otros colectivos o grupos de edad. Palabras clave: Motivación, ejercicio físico, educación superior, gestión deportiva, estudiante.

Abstract. This study aims to analyze the motivations toward physical activity of a representative sample of students from Universidad de Burgos, and their relationship with university sports services. 366 students (127 men and 239 women), whose average age was 21.29 years old, answered the validated scale on motives towards physical activity «Goal Content Exercise Questionnaire» (GCEQ), and another questionnaire about physical activity levels and participation in university sports service activities. Our empirical analysis determined that $48.6 \%$ of the students perform at least one physical activity weekly, the main motives being health care followed by skill development. Social aspects were not found to be significant. Less than a quarter of the students have used the university sports services. The lowest ranked aspect was compatibility of schedules. In conclusion, university students show low levels of physical activity, both within the college and outside of it. The motives towards the physical exercise do not show differences with other populations or age ranges.

Keywords: Motivation, physical exercise, college, sport management, student.

\section{Introducción}

Una gran parte de lapoblación española sigue sin incluir actividades físico-deportivas en su rutina habitual. En este sentido, las instituciones gubernamentales se encuentran con la necesidad de promocionar este tipo de prácticas desde la acción preventiva. En relación a la población joven, la legislación vigente cede gran parte de esta responsabilidad a las universidades, por considerarlas centros de desarrollo integral del estudiante (Martínez-Orga, 2006), que se rigen dentro de un marco legal demasiado impreciso entre la normativa educativa y la deportiva(Dopico, 2010), y que actúan a través de los servicios deportivos universitarios. Al igual que ocurre en los estudios con población general, los niveles de actividad física de los estudiantes universitarios españoles han determinado que la población activa durante este periodo oscila entre el 40\% y el 60\% (Arboleda, Arango, \& Feito, 2016; Castañeda-Vázquez \& Campos, 2012; Castillo-Viera \& Giménez, 2011), porcentaje ligeramente superior a los referenciados por estudios similares en otras regiones del mundo como Chile y Panamá (Durán, Castillo, \& Vio, 2009), México (Pérez-García, 2012), o Colombia (García, Herazo, \& Tuesca, 2015), pero preocupantes si consideramos que el comienzo de los estudios universitarios coincide con una disminución de los hábitos saludables de actividad físico-deportiva, lo que puede provocar a su vez el aumento de otros hábitos insalubres así como el comienzo de problemas de salud (Da Cuña, Lantarón, González, \& Gutiérrez, 2017; González \& Portolés, 2014).

Según Moral, Martínez y Grao (2013), los motivos que llevan a los estudiantes universitarios a realizar actividad física son similares a las de la población general. Entre otras, destacan para los estudiantes universitarios aquellos motivos relacionados con las actividades placenteras y que conlleven una implicación social y de amistad (Castañeda-Vázquez et al., 2014; Menéndez \& Fernández-Río, 2017), aunque depende del perfil de cada persona (Chacón et al., 2018). Igualmente, cabe destacar

Fecha recepción: 17-12-17. Fecha de aceptación: 31-07-18 Miguel Corbí Santamaría

mcorbi@ubu.es la motivación de los estudiantes hacia la actividad física por una mejora o mantenimiento de la salud, motivación que se mantiene en el tiempo una vez terminados los estudios universitarios (Almorza, Jiménez, \& Molero, 2011; Gómez-López, Ruiz, \& García, 2010), aunque cuando se trata de población enferma, la motivación hacia la actividad física suele ser bastante baja (Farholm \& Sorensen, 2016). Otras motivaciones muy recurrentes por los estudiantes universitarios son la competición, la capacidad personal, la aventura y la forma física asociada a la imagen corporal (Kilpatrick, Hebert, \& Bartholomew, 2005; PavónLores, Moreno-Murcia, Gutiérrez, \& Sicilia, 2003).

Aunque de manera no concluyente, algunos autores definen motivos de práctica físico-deportiva distintos entre hombres y mujeres, de modo que estas tendrían una tendencia mayor a relacionar la actividad física saludable con el mantenimiento de una buena imagen corporal y el control del peso (Castañeda-Vázquez et al., 2014; Kilpatrick et al., 2005; Tergerson \& King, 2002). A este respecto, los trabajos sobre los motivos de práctica físico-deportiva (Moreno-Murcia, González-Cutre, \& Cervelló, 2008) que se basan en el marco teórico de la Teoría de la Autodeterminación (Deci \& Ryan, 1985, 2000) ponen de manifiesto que la naturaleza intrínseca o extrínseca de dicha motivación define los patrones de conducta de la práctica de actividad física (Almagro, SaénzLópez, González-Cutre, \& Moreno-Murcia, 2011; Ednie \& Stibor, 2017). Según esta teoría, un practicante motivado intrínsecamente, practicará actividad físico-deportiva debido al disfrute que le proporciona esa actividad(Hassandra, Goudas, \& Chroni, 2003; Standage \& Treasure, 2002; Wang, Chatzisarantis, Spray, \& Biddle, 2002). De otro modo, la motivación extrínseca se basa en que el compromiso de la persona con la actividad se fundamenta en razones de tipo externo como, por ejemplo, practicar por conseguir un beneficio (Moreno-Murcia, MartínezGalindo, González-Cutre, \& Marcos, 2009).

El objetivo de este trabajo fue determinar aquellos factores que influyen en la práctica de actividad físico-deportiva de los estudiantes, diferenciando los niveles de práctica. Coincidimos con Canibe(2011)en que el deporte universitario debería estar dirigido hacia la mejora y mantenimiento de la salud y la calidad de vida, como una actividad formativa que contribuya a esa formación integral del estudiante. Para 
este fin, relacionar los datos obtenidos con el nivel de participación en las actividades propuestas por el servicio deportivo de la universidad permitirá la elaboración de estrategias de adherencia a la actividad físicodeportiva más eficaces y precisas.

\section{Material y métodos}

\section{Participantes}

La muestra estuvo formada por 366 estudiantes de grado de la Universidad de Burgos (UBU), 239 fueron mujeres (65.3\%) y 127, hombres (34.7\%), con edades comprendidas entre 17 y 49 años ( $M=$ 21.29; $\mathrm{DE}=4.03$ ). La muestra fue seleccionada de manera aleatoria y estratificada por centros universitarios (facultades de Ciencias, Ciencias de la Salud, Ciencias Económicas y Empresariales, Derecho, Educación, Humanidades y Comunicación, y Escuela Politécnica Superior), de modo que se trata de una muestra representativa de la UBU, con un nivel de confianza del $95 \%$ y un error muestral inferior a $\pm 5 \%$.

\section{Medidas}

Se utilizó la versión completa de la Goal Content for Exercise Questionnaire (GCEQ) de Sebire, Standage y Vansteenkiste (2008), validada al español por Moreno-Murcia, Marcos-Pardo y Huéscar (2016) para ser administrada tanto en población practicante como sedentaria. Esta escala consta de 20 ítems y cinco factores: afiliación social (i.e., «para hacer amigos y amigas»), imagen corporal (i.e., «para mejorar la apariencia y la forma de mi cuerpo»), reconocimiento social (i.e., "para ser bien considerado/a por otras personas»), salud (i.e., "para ser más resistente a la enfermedad») y desarrollo de las habilidad es (i.e., «para adquirir nuevas habilidades en el ejercicio físico»), que son los más habituales en la mayoría de estudios (Castillo-Viera \& Sáez-López, 2008). Estos ítems están encabezados por la frase «Indica en quémedida estos objetivos son importantes para ti en el ejercicio físico», teniendo que ser respondidos en una escala Likert de 1 (nada importante) a 7 (extremadamente importante). Los valores de alfa de Cronbach obtenidos en cada dimensión fueron: .91 para afiliación social; .88 para imagen corporal; .86 para reconocimiento social; .85 para salud; y .87 para el desarrollo de las habilidades. La forma de análisis de las respuestas se realiza por medio de los valores promedios obtenidos en cada dimensión.

Se incluyó un apartado de datos sociodemográficos, así como otro en el que se preguntaba si se practicaba alguna actividad físico-deportiva en la actualidad, si no se realizaba pero se había realizado en el pasado, o si nunca se había realizado ninguna actividad físico-deportiva más allá de las sesiones de Educación Física. Para precisar la información, se incluyó una pregunta acerca del tiempo dedicado en la actualidad y el pasado a 32 actividades deportivas propuestas y una de libre escritura. Además, se incluyó una pregunta acerca del uso del servicio deportivo universitario («¿Practicas o has practicado alguna actividad físico-deportiva a través de la unidad 'Deportes` de la UBU?») y donde se valoraba el grado de satisfacción en una escala de 0 (muy baja) a 5 (muy alta) de los siguientes aspectos: oferta de actividades, horario de las actividades, instalaciones, información de las actividades y compatibilidad con el horario de clases.

\section{Procedimiento}

Para el desarrollo de este estudio, se utilizó un diseño transversal por encuestas basado en la muestra. Se seleccionaron aleatoriamente grupos de estudio de diferentes facultades y escuelas superiores de la UBU. Una vez solicitado el permiso al Vicerrectorado de Investigación y Transferencia del Conocimiento de la UBU, se contactó con los profesores encargados de los grupos de alumnos para pedir su colaboración voluntaria. El investigador principal acudió a las aulas donde solicitó la participación de los estudiantes, explicó los objetivos y atendió todas las dudas que se plantearon. El tiempo necesario para su elaboración fue inferior a 10 minutos. El procedimiento fue aceptado por el Comité de Bioética de la UBU en informe con código IR 18/2018.

Una vez tratada la información recogida, la respuesta de los estu- diantes sobrepasó las necesidades de la muestra, obteniéndose un total de 394 cuestionarios completados correctamente. Se seleccionaron aleatoriamente los que finalmente formarían la muestra de 366, siempre teniendo en cuenta las proporciones de representación adecuadas por facultades y escuelas superiores. A partir de las respuestas se diferenciaron tres grupos a la hora de analizar los motivos: estudiantes practicantes (grupo «Practicantes»), definido como aquellos que realizaban, al menos, una actividad físico-deportiva semanalmente dentro o fuera del ámbito universitario, estudiantes sedentarios que abandonaron la práctica físico-deportiva en el pasado (grupo «Sedentarios I»), y estudiantes sedentarios que nunca realizaron actividad físico-deportiva de manera sistemática (grupo «Sedentarios II»).

\section{Análisis de los datos}

Una vez normalizados los datos obtenidos en los cuestionarios, se procedió al análisis de datos descriptivo e inferencial, realizados con el software SPSS Statistics (v.23.0), donde se consideraron significativas las asociaciones con un p-valor inferior a 0.05 . Para obtener los resultados sobre los efectos principales de la interacción entre las distintas variables se realizaron pruebas paramétricas univariantes (t de Student y ANOVA), en el caso de asociaciones entre variables cuantitativas entre dos grupos (Sedentarios vs. Practicantes) o tres grupos (Sedentarios I, Sedentarios II y Practicantes), y no paramétricas (Chi-cuadrado), en los casos de asociación entre variables cualitativas como el sexo de los participantes. Las variables cuantitativas mostraron tener una distribución normal. Tanto la escala GCEQ completa como sus dimensiones fueron sometidas al análisis de consistencia interna para determinar el coeficiente de fiabilidad de Cronbach.

\section{Resultados}

El 48.6\% de los encuestados afirmó practicar, al menos, una actividad física semanalmente, por lo que fueron designados como «Practicantes» (Tabla 1). El 43.2\% se definió como antiguo practicante («Sedentarios I») y solo el 8.2\% reportó no realizar ni haber realizado nunca alguna actividad físico-deportivo en el pasado («Sedentarios II»). Por sexo, los hombres mostraron porcentajes más favorables hacia la práctica de actividad física en la actualidad (64.6\% y 40.2\%, respectivamente), mientras que el porcentaje de mujeres que habían abandonado la práctica físico-deportiva en el pasado fue mayor al de los hombres (48.5\% de mujeres, por $33.1 \%$ de hombres). En cuanto a aquellos que no han practicado ninguna actividad físico-deportiva nunca, el porcentaje de mujeres (11.3\%) es sensiblemente mayor al de los hombres (2.4\%). La relación entre la práctica de actividad física y el sexo resultó significativa $(\tilde{n}<.001)$.

Realizados los análisis de varianza para establecer relaciones entre estos tres grupos y las cinco dimensiones que incluye el GCEQ (afiliación social, imagen, salud, reconocimiento social y desarrollo de las habilidades), las dos dimensiones en las que se observó una relación significativa son afiliación social y desarrollo de las habilidades (Tabla 1). El conjunto de «Sedentarios II» muestra una puntuación inferior a los otros dos grupos en la dimensión afiliación social. Por otro lado, el grupo de «Practicantes» muestra una mayor afinidad hacia el desarrollo de las habilidades que los dos grupos sedentarios. La prueba de comparación múltiple de Bonferroni para ambas dimensiones determinó que la dimensión relacionada con el desarrollo de las habilidades se observa una tendencia significativa hacia puntuaciones más altas en el grupo de practicantes que en cualquiera de los grupos de sedentarios $(\tilde{n}<.01)$. Por su parte, el grupo de sedentarios que nunca ha realizado actividad física tiende significativamente hacia puntuaciones más bajas en los ítems relacionados con la dimensión de afiliación social $(\tilde{n}<.05)$ quelos otros dos grupos.

Las puntuaciones obtenidas por el grupo «Practicantes» revelan que el principal motivo de este grupo hacia la actividad física es la salud $(M=5.45)$, seguido por el desarrollo de las capacidades. El motivo que menos puntuación obtuvo, con una gran diferencia es el reconocimiento social $(M=2.16)$, mientras que la afiliación social y la imagen son 
Tabla 1.

\begin{tabular}{|c|c|c|c|c|c|c|}
\hline & $N$ & $\begin{array}{c}\text { Afil. Social } \\
M+D E\end{array}$ & $\begin{array}{l}\text { Imagen } \\
M+D E\end{array}$ & $\begin{array}{c}\text { Salud } \\
M+D E\end{array}$ & $\begin{array}{l}\text { Rec. Social } \\
M+D E\end{array}$ & $\begin{array}{l}\text { Des. Habilidades } \\
M+D E\end{array}$ \\
\hline Practicantes & 178 & $3.7^{*} \pm 1.6$ & $4.0 \pm 1.4$ & $5.5 \pm 1.1$ & $2.2 \pm 1.2$ & $4.8^{* *} \pm 1.4$ \\
\hline Sedentarios I & 158 & $3.7 * \pm 1.5$ & $4.1 \pm 1.5$ & $5.4 \pm 1.1$ & $2.4 \pm 1.3$ & $4.4 * * \pm 1.5$ \\
\hline Sedentarios II & 30 & $2.8^{*} \pm 1.4$ & $4.0 \pm 1.6$ & $5.3 \pm 1.4$ & $1.9 \pm 1.1$ & $3.98^{* *} \pm 1.5$ \\
\hline \multicolumn{7}{|c|}{ Nota: ${ }^{*} p<0.05 ;{ }^{* *} p<0.01$} \\
\hline \multicolumn{7}{|c|}{$\begin{array}{l}\text { Tabla } 2 \text {. } \\
\text { Puntuaciones medias y desviaciones en las valoraciones acerca de diferentes aspectos } \\
\text { relacionados con el servicio deportivo universitario, total y por sexo. }\end{array}$} \\
\hline & & \multirow{2}{*}{\multicolumn{2}{|c|}{$\begin{array}{c}\text { Total } \\
M \pm D E\end{array}$}} & \multicolumn{2}{|c|}{ Hombres } & Mujeres \\
\hline & & & & \multicolumn{2}{|c|}{$M \pm D E$} & $M \pm D E$ \\
\hline Oferta & & \multicolumn{2}{|c|}{$3.0 \pm 1.3$} & \multirow{2}{*}{\multicolumn{2}{|c|}{$\begin{array}{l}3.1 \pm 1.3 \\
2.4 \pm 1.4\end{array}$}} & $2.9 \pm 1.4$ \\
\hline Horario & & \multicolumn{2}{|c|}{$2.2 \pm 1.5$} & & & $2.1 \pm 1.5$ \\
\hline Instalaciones & & \multicolumn{2}{|c|}{$2.7 \pm 1.3$} & \multicolumn{2}{|c|}{$2.9 \pm 1.3$} & $2.6 \pm 1.3$ \\
\hline Información & & \multicolumn{2}{|c|}{$2.7 \pm 1.4$} & \multicolumn{2}{|c|}{$2.9 \pm 1.4$} & $2.6 \pm 1.5$ \\
\hline Compatibilidad & & \multicolumn{2}{|c|}{$1.8 \pm 1.6$} & \multicolumn{2}{|c|}{$2.0 \pm 1.6$} & $1.7 \pm 1.6$ \\
\hline
\end{tabular}

consideradas con puntuaciones intermedias. En ambos grupos sedentarios, la dimensión que obtuvo una valoración media más alta fue la salud ( $M=5.36)$, seguido del desarrollo de las habilidades y la imagen. La dimensión con menor puntuación media resultó ser el reconocimiento social $(M=2.31)$.

En el caso de los grupos sedentarios no se han observado diferencias significativas entre ellos, aunque sí existe una diferencia significativa entre las medias obtenidas por ambos grupos en relación a la dimensión de afiliación social $(\tilde{n}=.008)$. El valor obtenido por los «Sedentarios I» ( $M=3.65 ; D E=1.53)$ fue significativamente mayor al valor resultante del grupo «Sedentarios II» $(M=2.83 ; D E=1.44)$.

El 23.2\% de los encuestados reportó practicar o haber practicado alguna de las actividades con el servicio deportivo universitario, siendo los hombres quienes han utilizado o utilizan este servicio con mayor asiduidad que las mujeres (38.6\% de hombres y 15.1\% de mujeres). La evaluación del grado de satisfacción de estos estudiantes con respecto al servicio deportivo universitario (Tabla 2) mostró valoraciones promedio intermedias, siendo el aspecto más valorado la oferta de actividades ( $M=2.97)$ y la menos valorada la compatibilidad de los horarios de las actividades con los horarios de clase $(M=1.82)$. No se observó significatividad en la relación entre las medias obtenidas y el sexo de los participantes $(\tilde{n}>.05)$.

\section{Discusión}

Los resultados han identificado algunas relaciones significativas entre los diferentes grupos según nivel de actividad físico-deportiva. En relación a los motivos acerca del desarrollo de las habilidades físicas, el grupo de practicantes obtiene valoraciones más altas que los grupos de sedentarios, lo que coincide con los resultados de Capdevila, Niñerola y Pintanel (2004). En los motivos referidos a la afiliación social y al contacto con otras personas, el grupo de sedentarios que nunca realizó actividad física evidenció diferencias con respecto a los otros dos grupos, obteniendo puntuaciones notablemente más bajas. Estos resultados coinciden con los obtenidos por Ribeiro, Ferreira, Sampaio y Alchieri (2015) en un estudio con una muestra de universitarios brasileños, y con los obtenidos por Moreno-Murcia, Cervelló y Martínez (2007) sobre población general en España. En ambos casos se utilizó el cuestionario MPAM-R validado por Ryan, Frederick, Lepes, Rubio y Sheldon (1997), que cuenta con unas dimensiones y escalas similares a las utilizadas en nuestra investigación. Los resultados obtenidos en estos estudios ponen de manifiesto que la salud es uno de los principales motivos para la realización de actividad física, tanto para las personas activas como para las sedentarias. Estos resultados coinciden en parte con el estudio de Moreno-Murcia et al. (2016), donde encuentran una relación positiva en el grupo de practicantes entre los motivos de afiliación, reconocimiento social y desarrollo de las habilidades, aunque en este caso solo se trató con una muestra de mujeres. Coincidiendo con los resultados de Blázquez y Feu (2012), nuestros resultados acerca de los motivos de las mujeres practicantes apoyan la ruptura del estereotipo atribuido a estas, al no relacionar imagen con afiliación social ni con desarrollo de las habilidades.

El principal motivo que encuentran los estudiantes físicamente activos es el cuidado de la salud. Estos resultados han sido similares con otros en los que, además de la salud, también se obtuvieron valores muy positivos en los motivos relacionados con la diversión(Alonso \& García, 2010; Castañeda-Vázquez \& Campos, 2012; Moreno-Murcia et al., 2016). La relación entre actividades físico-deportivas y la salud es de sobra conocida por los estudiantes universitarios. El estudio de Haase, Steptoe, Sallis y Wardle (2004), donde compararon 23 países de todo el mundo, sitúa a España en una posición intermedia en cuanto al conocimiento de la relación actividad físico-deportiva y salud. Países como Eslovaquia, Japón y Grecia son los que demuestran puntuaciones mayores en este sentido, pero curiosamente son países que no reportaron altos niveles de actividad físico-deportiva entre sus universitarios. Estos datos permiten observar cómo, a pesar de que el motivo de salud es uno de los más recurridos a la hora de incentivar la práctica de actividad físico-deportiva, el nivel de participación no depende del hecho de reconocer la actividad físico-deportiva como instrumento de salud.

El segundo motivo que ha obtenido la puntuación más alta en nuestros resultados hace referencia al desarrollo de las capacidades, y el tercero, a la imagen. Estos resultados no han mostrado diferencias significativas entre sexos, a pesar de que tradicionalmente se han atribuido las motivaciones relacionadas con el desarrollo de las capacidades a los hombres y las relacionadas con el cuidado de la imagen a las mujeres. No obstante, existen otras causas, además del sexo, que han demostrado relación con las motivaciones hacia la mejora de la imagen. En este sentido, un artículo de Guedes, Legnani y Legnani (2013), determinó que existe una relación positiva entre la motivación hacia la imagen y el nivel socioeconómico de los universitarios, por lo que tanto hombres como mujeres con un nivel adquisitivo elevado pueden verse influidos por esta motivación. Nuestros resultados coinciden con aquellos en los que no ha existido una diferencia significativa entre hombres y mujeres (Gómez-López, 2005; Madanat \& Merril, 2006), pero difieren de aquellos otros en los que se ha observado que las mujeres tienen una especial predilección por las motivaciones relacionadas con la imagen (Fernández-Ozcorta, Sáenz-López,Almagro, \& Conde, 2015; Guedes et al., 2013; Kilpatrick et al., 2005). De hecho, en nuestro caso, son los hombres los que han obtenido puntuaciones más altas en los motivos relativos al cuidado de la imagen, posiblemente debido a la influencia de los cánones estéticos actuales (Moreno-Murcia et al., 2016).

Otro de los motivos que incentivan la práctica físico-deportiva es la búsqueda de algún tipo de reconocimiento social, que se ha atribuido generalmente a los hombres. El interés por demostrar el valor, comparar capacidades, alcanzar grandes logros o ganarse el reconocimiento de los demás, pueden ser algunos de los argumentos que inciten a los jóvenes a la práctica de actividades físico-deportivas (Markland \& Ingledew, 1997). En la actualidad, dados los valores sociales que se vierten a partir de los medios de comunicación, una motivación relacionada con la imagen también está relacionada, de algún modo, con los motivos sociales de reconocimiento y afiliación. Principalmente en el pasado, pero también ocurre en la actualidad, la mujer se ha visto sometida a una gran presión social procedente de la preocupación por mantener una apariencia física determinada, relacionada con la necesidad de valoración positiva por parte de la sociedad (Moreno-Murcia et al., 2016). Aunque la relación no ha sido significativa, y teniendo en cuenta que los participantes en nuestro estudio han obtenido puntuaciones bajas en relación al reconocimiento social a partir de la actividad físico-deportiva, nuestros resultados coinciden con Kilpatrick et al. (2005) y García et al. (2015), quienes determinaron que las mujeres presentaban tasas más bajas que los hombres. Por otro lado, la dimensión afiliación social también ha obtenido valores bajos, siendo la puntuación de las mujeres inferior a la de los hombres. Este resultado está en línea con otras investigaciones, puesto que, aunque algunos trabajos como el de Gill, Dowd, Beaudoin y Martin (1996) han manifestado la importancia del motivo de afiliación para la participación en las actividades físico-deportivas de las mujeres, la mayoría de estudios atribuyen las motivaciones sociales al hombre (Moreno-Murcia et al., 2016).

Puede destacarse que, entre los grupos de sedentarios, aquellos que nunca practicaron actividad física presentan valores inferiores en cuanto a la afiliación social. Es decir, no supone para ellos un motivo utilizar 
estas actividades como vía de relación social, cuestión que aquellos que abandonaron la práctica de actividad físico-deportiva en el pasado sí valoran. En relación a los motivos con puntuaciones más altas, los grupos sedentarios estudiados afirmaron valorar el aspecto de salud como el más importante dentro de los motivos que subyacen a la práctica de actividad física regular. Los motivos que les siguen en importancia son el desarrollo de las habilidades y las relacionadas con el cuidado de la imagen. Ambos grupos coinciden en otorgar la menor importancia a los motivos que tienen relación con el reconocimiento social. Al igual que ocurría con el grupo de practicantes, las mujeres sedentarias otorgan menor importancia que los hombres a los motivos relativos al ámbito social (afiliación social y reconocimiento social). Es decir, su manera de interrelación con sus iguales no suele estar determinada por la práctica de actividades físico-deportivas.

El porcentaje de estudiantes que realiza o ha realizado algún tipo de actividad físico-deportiva con el servicio deportivo de la Universidad se sitúa en el 23.2\%, siendo mayor el porcentaje de hombres (38.6\%) que el de mujeres (15.1\%). Este resultado, mayor que el encontrado en el estudio de Castañeda-Vázquez, Romero-Granados y Ríes (2012), puede deberse a que los hombres son más participativos que las mujeres en las actividades de naturaleza competitiva que, aunque no suponen una mayoría clara en la oferta de la Universidad, suelen ser más llamativas y facilitan el acceso a los estudiantes a competiciones que de otra manera no podrían acceder.

En relación a los diferentes aspectos evaluados sobre el servicio deportivo universitario, las puntuaciones atribuidas por nuestra muestra han obtenido valores intermedios, siendo lo mejor valorado la oferta de actividades (2.97 sobre 5), aunque esta valoración es ligeramente superior en el caso de los hombres (3.11 sobre 5) que en el de las mujeres (2.89 sobre 5). Los aspectos peor valorados son los referidos a los horarios. De este modo, el aspecto con menor puntuación ha resultado ser la compatibilidad de horarios con las horas de clase (1.82 sobre 5), seguido del horario de las actividades (2.22 sobre 5). Como han determinado otros estudios (De Mena-Ramos, 2015; Flores-Allende, 2009; Gómez-López, 2005), la incompatibilidad de los horarios con el resto de obligaciones de los estudiantes es una de las principales causas de abandono entre la población universitaria, que generalmente encuentra insatisfechas sus necesidades físico-deportivas de cualquier nivel en el ámbito universitario (Blanco \& Burillo, 2018).

Consideramos que la información acerca de los servicios que ofrecen los servicios deportivos universitarios de las universidades es uno de los pilares de la participación en las actividades ofertadas. El informe del Consejo Superior de Deportes (2006) sobre el deporte universitario español concluye que el 23\% de los universitarios que son asiduos en la práctica de alguna actividad físico-deportiva no conoce los programas deportivos ofertados por sus universidades. Este valor es más bajo que los obtenidos por diferentes investigadores como Alonso y García (2010) o Castañeda-Vázquez et al. (2012), quienes no superan el 40\%, pero notablemente más bajo que el reciente resultado de De Mena-Ramos (2015), quien obtuvo un porcentaje superior al $55 \%$ de desinformación. En nuestro caso, el 39.9\% de los encuestados afirma que la información recibida es satisfactoria, mientras que el $27.6 \%$ la considera insatisfactoria y el porcentaje restante (32.5\%) no sabe o no contesta a esta pregunta. Los resultados obtenidos son sensiblemente mejores que los mostrados en otros estudios donde el porcentaje de satisfacción es inferior al 12\% (De Mena-Ramos, 2015), o el porcentaje de insatisfacción es superior al 35\% (Consejo Superior de Deportes, 2006).

En lo relativo a la oferta de actividades, García-Ferrando y Llopis (2011) establecen un índice del 32.6\% de insatisfacción de la población española hacia la oferta de las instituciones responsables de la promoción deportiva. Según nuestros resultados, encontramos una relación significativa entre el sexo y la satisfacción con la oferta. Los hombres se encuentran satisfechos en un 56.7\%, mientras que solo el 36.8\% de las mujeres admite encontrarse satisfecha con las actividades propuestas por el servicio universitario. Posiblemente los deportes de competición, más atractivos para hombres que para mujeres, sean la parte más visible entre todas las actividades propuestas por los diferentes servicios de- portivos universitarios. Las características de organización y la expectativa que promueve la competición, unida a la celebración de eventos como los Campeonatos de España Universitarios, favorecen la difusión de este tipo de actividades por encima de aquellas que están relacionadas con el deporte-salud o entretenimiento. No obstante, es posible que la comunidad universitaria, bien por desconocimiento o por insatisfacción real, tenga una tendencia a solicitar a los servicios deportivos universitarios la presencia de más actividades físico-deportivas no competitivas (Castañeda-Vázquez et al., 2012).

Entre las limitaciones de este estudio, destacamos que el uso de la escala GCEQ, si bien es una escala que permite medir los motivos de práctica físico-deportiva tanto de población practicante como sedentaria, únicamente valora motivos de origen extrínseco, por lo que, según la Teoría de Autodeterminación de Deci y Ryan $(1985,2000)$ no evalúa todo el espectro de la motivación, que se compone de motivación intrínseca, extrínseca y amotivación. Esta última, en este caso, medida como ausencia de motivación. Por otro lado, otras variables socioculturales, como el clima motivacional, el apoyo social y/o familiar o la relación con los instructores, puedan afectar sobre la adherencia a la actividad físico-deportiva de nuestros estudiantes que no han sido contempladas en este estudio, por lo que sería recomendable contar con investigaciones de carácter cualitativo que dé voz a todos los participantes por medio de grupos de discusión que incluyan tanto personas expertas como inexpertas, para profundizar en los modos que deben incluir las estrategias de promoción deportiva en el ámbito universitario.

\section{Conclusiones}

Independientemente del nivel de práctica físico-deportiva, el primer motivo de los estudiantes para llevarla a cabo es la atención de la salud, seguido del desarrollo de las habilidades físicas y los cuidados con la imagen, sin diferencias significativas entre sexos. Para los estudiantes que no practican y tampoco han practicado actividad físico-deportiva en el pasado, la afiliación social no es motivo para la realización de actividades físico-deportivas.

Menos de un cuarto de los estudiantes practica o ha practicado alguna actividad física-deportiva con el servicio deportivo de la Universidad, siendo más propensos los hombres que las mujeres. La principal barrera percibida para la participación es la incompatibilidad de los horarios de las actividades con los horarios de las clases.

\section{Referencias}

Almagro, B. J., Saénz-López, P., González-Cutre, D., \& Moreno-Murcia, J. A. (2011). Clima motivacional percibido, necesidades psicológicas y motivación intrínseca como predictores del compromiso deportivo en adolescentes. Revista Internacional de Ciencias del Deporte, 25(7), 250-265.

Almorza, D., Jiménez, J. A., \& Molero, J. (2011). Estudio diagnóstico del deporte universitario español. Madrid: Consejo Superior de Deportes.

Alonso, D., \& García, J. L. (2010). Motivación hacia la práctica físicodeportiva de los universitarios gallegos. Revista de Investigación en Educación, 8, 128-138.

Arboleda, V. H., Arango, E. F., \& Feito, Y. (2016). Actividad física y percepciones de beneficios y barreras en una universidad colombiana. Retos, 30, 15-19.

Blanco, P., \& Burillo, P. (2018). Los deportistas de élite en el sistema universitario español. Retos(33), 162-168.

Blázquez, A., \& Feu, S. (2012). Motivación en actividad física mantenimiento en mujeres de municipios pequeños. Revista Internacional de Medicina y Ciencias de la Actividad Física y el Deporte, 12(47), 571-588.

Canibe, A. (2011). El papel del deporte en los campus de excelencia universitarios. Tándem, 38-46.

Capdevila, L., Niñerola, J., \& Pintanel, M. (2004). Motivación y actividad física: el autoinforme de motivos para la práctica de ejercicio físico (AMPEF). Revista de Psicología del Deporte, 13(1), 55-74.

Castañeda-Vázquez, C., \& Campos, M. C. (2012). Motivación de los estudiantes de la Facultad de Ciencias de la Educación (Universidad de 
Sevilla) hacia la práctica de actividad físico-deportiva. Retos, 22, 5761.

Castañeda-Vázquez, C., Romero-Granados, S., \& Ríes, F. (2012). Práctica deportiva y opinión del alumnado sobre el Servicio de Actividades Deportivas en la Facultad de Ciencias de la Educación (Universidad de Sevilla). Revista de Investigación en Educación, 10(1), 60-75.

Castañeda-Vázquez, C., Zagalaz, M. L., Chacón, F., Cachón, J., \& Romero, S. (2014). Características de la práctica deportiva en función del género. Estudiantes de la Facultad de Ciencias de la Educación: Universidad de Sevilla. Retos, 25, 63-67.

Castillo-Viera, E., \& Giménez, F. J. (2011). Hábitos de práctica de actividad física del alumnado de la Universidad de Huelva. Revista Internacional de Medicina y Ciencias de la Actividad Física y el Deporte, 10(41), 127-144.

Castillo-Viera, E., \& Sáez-López, P. (2008). Práctica de actividad física y estilo de vida del alumnado de la Universidad de Huelva. Huelva: Servicio de Publicaciones de la Universidad de Huelva.

Chacón, R., Chacón, F., Zurita, F., Cachón, J., Zagalaz, M. L., \& Chinchilla, J. J. (2018). Characterization of motivation and type of physicalsport practice in adults through COMPASS profiles. Journal of Human Sport \& Exercise, 13(1), 161-173. doi:https://doi.org/10.14198/ jhse.2018.131.16

Consejo Superior de Deportes. (2006). El Modelo del Deporte Universitario Español. Córdoba: Universidad de Córdoba.

Da Cuña, I., Lantarón, E. M., González, Y., \& Gutiérrez, M. (2017) Repercusión del sedentarismo en la respuesta cardiorrespiratoria en estudiantes universitarios. Revista Internacional de Medicina y Ciencias de la Actividad Física y el Deporte, 17(66), 367-378. doi:https:/ /doi.org/10.15366/rimcafd2017.66.010

De Mena-Ramos, J. M. (2015). Motivaciones, hábitos e intereses hacia la práctica de actividad físico deportivas de los estudiantes de la Universidad Pontificia de Salamanca. Tesis Doctoral. Salamanca: Universidad Pontificia de Salamanca. Facultad de Educación.

Deci, E. L., \& Ryan, R. M. (1985). The general causality orientations scale: Self-determination in personality. Journal of Research in Personality, 19, 109-134.

Deci, E. L., \& Ryan, R. M. (2000). The «what» and «why» of goal pursuits: Human needs and the self-determination of behaviour Psychological Inquiry, 11, 227-268.

Dopico, X. (2010). El deporte universitario y su reconocimiento académico. 10th FIFU Forum. University Sport: a platform for change. Vigo: International University Sport.

Durán, S., Castillo, M., \& Vio, F. (2009). Diferencias en la calidad de vida de estudiantes universitarios de diferente año de ingreso del Campus Antumapu. Rev. Chlil. Nutr., 36(3), 200-209.

Ednie, A., \& Stibor, M. (2017). Influence and interpretation of intrinsic and extrinsic exercise motives. Journal of Human Sport and Exercise, 12(2), 414-425. doi:10.14198/jhse.2017.122.18

Farholm, A., \& Sorensen, M. (2016). Motivation for physical activity and exercise in severe mental illness: A systematic review of intervention studies. International Journal of Mental Health Nursing, 25, 194-205.

Fernández-Ozcorta, E. J., Sáenz-López, P., Almagro , B. J., \& Conde, C. (2015). Motivos de práctica de actividad física en universitarios activos. Revista Digital de Educacaión Física EmásF, 34, 52-60.

Flores-Allende, G. (2009). Actividad físico-deportiva del alumnado de la universidad de Guadalajara (México). Correlatos biológicos y cognitivos asociados (Tesis Doctoral). Murcia: Universidad de Murcia.

García, F., Herazo, Y., \& Tuesca, R. (2015). Factores sociodemográficos y motivacionales asociados a la actividad física en estudiantes universitarios. Revista Médica de Chile, 143, 1411-1418.

García-Ferrando, M., \& Llopis, R. (2011). Ideal democrático y bienestar personal. Encuesta sobre hábitos deportivos en España 2010. Madrid: Consejo Superior de Deportes.

Gill, D., Dowd, D. A., Beaudoin, C. M., \& Martin, J. (1996). Competitive orientation and motives of adults sport and exercise participants. Journal of Sport Behavior, 19, 307-318.

Gómez-López, M. (2005). La actividad físico-deportiva en los centros almerienses de educación secundaria post-obligatoria y en la Universidad de Almería. Evolución de los hábitos físico-deportivos de su alumnado (Tesis Doctoral). Almería: Universidad de Almería.

Gómez-López, M., Ruiz, F., \& García, M. E. (2010). Actividades físicodeportivas que demandan los universitarios. Espiral. Cuadernos del Profesorado, 3(5), 3-10.
González, J., \& Portolés, A. (2014). Actividad física extraescolar: Relaciones con la motivación educativa, rendimiento académico y conductas asociadas a la salud. Revista Iberoamericana de Psicología del Ejercicio y el Deporte, 9(1), 51-65.

Guedes, D. P., Legnani, R. F., \& Legnani, E. (2013). Exercise motives in a sample of Brazilian university students. Motriz, Río Claro, 19(3), 590-596.

Haase, A., Steptoe, A., Sallis, J. F., \& Wardle, J. (2004). Leisure-time physical activity in university students from 23 countries: associations with health beliefs, risk awarness, and national economic development. Preventive Medicine, 39, 182-190.

Hassandra, M., Goudas, M., \& Chroni, S. (2003). Examining factors associated with intrinsic motivation in physical education: a qualitative approach. Psychology of Sport and Exercise, 4, 211-223.

Kilpatrick, M., Hebert, E., \& Bartholomew, J. (2005). College students' motivation for physical activity: Differentiating men's and women's motives for sport participation and exercise. Journal of American College Health, 54, 87-94.

Madanat, H., \& Merril, R. (2006). Motivational factors and stages of change for physical activity among college students in Amman, Jordan. Promotion \& Education, 13, 185-190.

Markland, D., \& Ingledew, D. K. (1997). The measurment of exercise motives: Factorial validity and invariance across gender of a revised Exercise Motivation Inventory. British Journal of Health Psychology, 2, 361-376.

Martínez-Orga, V. (2006). Orientación y tendencias del deporte universitario en España. En R. Terol, El deporte universitario en España: actualidad y perspectivas de futuro (págs. 173-184). Madrid: Dykinson.

Menéndez, J. I., \& Fernández-Río, J. (2017). Responsabilidad social, necesidades psicológicas básicas, motivación intrínseca y metas de amistad en Educación Física. Retos(32), 134-139.

Moral, J. E., Martínez, E., \& Grao, A. (2013). Sedentarismo, salud e imagen corporal en adolescentes. Sevilla: Wanceulen.

Moreno-Murcia, J. A., Cervelló, E., \& Martínez, A. (2007). Validación de la escala de medida de los Motivos para la Actividad Física-Revisada en españoles: Diferencias por motivos de participación. Anales de Psicología, 23(1), 167-176.

Moreno-Murcia, J. A., González-Cutre, D., \& Cervelló, E. (2008). Motivación y salud en la práctica físico-deportiva: diferencias según el consumo de alcohol y tabaco. International Journal of Clinical and Health Psychology, 8(2), 483-494.

Moreno-Murcia, J. A., Marcos-Pardo, P. J., \& Huéscar, E. (2016). Motivos de práctica físico-deportiva en mujeres: diferencias entre practicantes y no practicantes. Revista de Psicología del Deporte, 25(1), 35-41.

Moreno-Murcia, J. A., Martínez-Galindo, C., González-Cutre, D., \& Marcos, P. (2009). Perfiles motivacionales de practicantes en el medio acuático frente al medio terrestre. Revista Internacional de Medicina y Ciencias de la Actividad Física y el Deporte, 9(34), 201216.

Pavón-Lores, A., Moreno-Murcia, J. A., Gutiérrez, M., \& Sicilia, A. (2003). La práctica físico-deportiva en la Universidad. Revista de Psicología del Deporte, 12(1), 39-54.

Pérez-García, J. A. (2012). Motivaciones y etapas de cambio de comportamiento ante la actividad físico-deportiva en estudiantes de la Universidad Autónoma de Nuevo León (México) (Tesis Doctoral). Sevilla: Universidad Pablo de Olavide.

Ribeiro, S. M., Ferreira, R., Sampaio, A., \& Alchieri, J. C. (2015). Motivation and self-esteem in university students' adherence to physical activity. Revista de Salud Pública, 677-688.

Ryan, R. M., Frederick, C. M., Lepes, D., Rubio, N., \& Sheldon, K. M. (1997). Intrinsic motivation and exercise adherence. International Journal of Sport Psychology, 28, 335-354.

Standage, M., \& Treasure, D. (2002). Relationship among achievement goal orientations and multidimensional situational motivation in physical education. British Journal of Education Psychology, 72, 87103.

Tergerson, J. L., \& King, K. A. (2002). Do perceived cues, benefits and barriers to physical activity differ between male and female adolescents? Journal of School Health, 72(9), 374-380.

Wang, C. K., Chatzisarantis, N., Spray, C., \& Biddle, S. (2002). Achievement goal profile in school physical education: Differences in self-determination, sport ability beliefs, and physical activity. British Journal of Educational Psychology, 72, 433-445. 